\section{PTH-024 SELF PROVIDED GUIDED MEDICAL HISTORY IS FAST, COMPLETE AND ACCURATE}

${ }^{1} \mathrm{C}$ Van Someren*, ${ }^{2} \mathrm{~N}$ van Someren, ${ }^{1} \mathrm{~A}$ Millar, ${ }^{1} \mathrm{G}$ Edwards. 'Gastroenterology, North Middlesex University Hospital, London, UK; ${ }^{2}$ Gastroenterology, Chase Farm Hospital, London, UK

\subsection{6/gutjnl-2014-307263.470}

Introduction The medical history is the fundamental basis of diagnosis. Every medical student learns how to take a full history, but in clinical practice the history is often truncated due to pressures of various kinds. Many clinicians use pre-consultation forms to collect relevant history, but these are rigid and cannot include the complete medical history.

Methods We have developed a large database of questions that could be asked during history taking. A computerised algorithm selects the next relevant question depending on the previous answer. A designer interface allows questionnaires to be developed and adjusted readily. The questions are phrased in plain English, but the program translates the answers into medical terminology. The history is then available in PDF format for presentation to the clinician. Patients self completed their history using a touch screen, and checked the results before printing. Comments about the process were recorded from patients and clinical staff. The system was trialled in a hepatitis assessment clinic. Patients are fully informed about this voluntary and secure system prior to use.

Results 443 patients used the touch screen. 12 did not complete their history because of language problems (8) or indifference (3). The average time to complete was 14.7 min (range 7-21 min). 7 patients were identified who were at high risk of hepatitis infection, and a monospot test was offered and accepted in all of these and further serological tests undertaken. 180 patients with known positive serology completed their history.

Conclusion Patients found the touch screen easy to use, and were able to complete their history in the waiting area prior to consultation. They were universally happy to keep a printout of their history. The clinicians were able to spend more time discussing risks and treatment options, and were able to ask supplementary questions rather than repeatedly obtaining basic data. Printouts of the PDF were retained in the notes as part of the medical record. This technology has shown great potential in allowing more new patients to be seen, increasing efficiency in carrying out regular reviews, gathering better clinical information and reducing patient distress when asking sensitive questions. Perceived benefits are - more rapid and thorough clinical assessment; semi-automated follow up; health screening; and patient surveys. There are applications for this technology in many fields of medical practice.

Disclosure of Interest None Declared.

\section{PTH-025 DIETITIAN-LED VIRTUAL COELIAC DISEASE CLINIC RESULTS IN COST SAVINGS AND INCREASED PATIENT SATISFACTION}

${ }^{1} \mathrm{C}$ Stuckey*, ${ }^{1} \mathrm{H}$ Martin, ${ }^{2} \mathrm{~S}$ McLaughlin. ${ }^{1}$ Nutrition and Dietetics, Royal Bournemouth Hospital, Bournemouth, UK; '2Gastroenterology, Royal Bournemouth Hospital, Bournemouth, UK

\subsection{6/gutjnl-2014-307263.471}

Introduction We identified that demand for follow up of adult patients diagnosed with coeliac disease exceeded the capacity of our dietitian-led coeliac annual review clinic. A dietitian-led virtual coeliac clinic was developed as an efficient and cost effective method of reviewing adults with established coeliac disease. Methods Patients with established coeliac disease (i.e. a combined histological and serological diagnosis more than 12 months earlier), who were assessed by the dietitian as understanding fully how to adhere to a gluten free diet and who have shown a good symptomatic response were sent the virtual clinic questionnaire. They were also sent a blood form to monitor full blood count, bone biochemistry, ESR, haematinics and tissue transglutaminase in line with the BSG 2010 coeliac guidelines. They were asked to complete and return the questionnaire in a prepaid addressed envelope and have the blood tests undertaken within 4 weeks. The questionnaire responses and blood results were reviewed by a specialist gastroenterology dietitian. The identification of any new abnormal blood results was highlighted to the GP and/or consultant gastroenterologist. If any red flag symptoms (i.e. blood in stools, unintentional weight loss or persistent change in bowel habit) were highlighted on the questionnaire then the patient was telephoned by the dietitian to discuss further before being referred to their GP or gastroenterologist as appropriate.

Results 86 patients were sent the virtual coeliac review questionnaire from May 2012- April 2013. Of these, 81 patients (94.2\%) returned their questionnaire and 78 patients $(90.6 \%)$ had their blood tests undertaken. Of these, 7.4\% patients $(\mathrm{n}=$ 6) were referred to gastroenterology for abnormal blood results or for the presence of new gastrointestinal symptoms. Only 1 of these patients $(1.2 \%)$ needed to be seen in the gastroenterology clinic. $6.1 \%$ of patients $(n=5)$ were telephoned by the dietitian for reporting red flag symptoms. After the telephone consultation none of these patients required gastroenterology or dietetic appointments.

$80 \%$ of patients responded that alternating annually between virtual review and a clinic appointment was preferential to an annual clinic appointment.

Conclusion A virtual review coeliac clinic at the Royal Bournemouth Hospital is a cost effective and patient preferred method of managing adults with established coeliac disease.

\section{REFERENCE}

1 The Management of Adults with Coeliac Disease; BSG 2010

Disclosure of Interest None Declared.

\section{PTH-026 EVALUATION OF A SERVICE TO MANAGE INFLAMMATORY BOWEL DISEASE (IBD) IN PREGNANCY}

C Parker*, M Gunn. Gastroenterology, Royal Victoria Informary, Newcastle Upon Tyne, UK

\subsection{6/gutjnl-2014-307263.472}

Introduction Following the publication of a consensus for management of IBD in pregnancy a service was set up in our institution (April 2013) to optimise the management of pregnant women with IBD. Its' aim is to enable multidisciplinary management of patients with a consultant gastroenterologist, consultant obstetrician, colorectal surgeon and IBD specialist nurse. It provides a baseline health check in the early stages of pregnancy, more intensive foetal growth monitoring with additional growth scans at 28 and 32 weeks of pregnancy, discussion of delivery methods and anticipation of potential peripartum problems including liaison with colorectal surgical team. A review of the service was performed after 8 months to provide an overview of management of patients, demand and outcomes following set up of the service. 